\title{
A REMARK ON THE INTERSECTION OF THE CONJUGATES OF THE BASE OF QUASI-HNN GROUPS
}

\author{
R. M. S. MAHMOOD
}

Received 25 September 2003

Quasi-HNN groups can be characterized as a generalization of HNN groups. In this paper, we show that if $G^{*}$ is a quasi-HNN group of base $G$, then either any two conjugates of $G$ are identical or their intersection is contained in a conjugate of an associated subgroup of $G$.

2000 Mathematics Subject Classification: 20F65, 20E07, 20 E08.

1. Introduction. In [8, Lemma 3.15, page 152], Scott and Wall proved that if $G=$ $G_{1} *_{C} G_{2}$ is a nontrivial free product with amalgamation group, then either $g G_{1} g^{-1} \cap G_{i}$ is a subgroup of a conjugate of $C$, or $i=1$ and $g \in G_{1}$, so that $g G_{1} g^{-1} \cap G_{i}=G_{1}$. In this paper we generalize such a result to groups acting on trees with inversions and then apply the result we obtain to a new class of groups called quasi-HNN groups, introduced in [2]. This paper is divided into five sections. In Section 2, we give basic definitions. In Section 3, we have notations related to groups acting on trees with inversions. In Section 4 , we discuss the intersections of vertex stabilizers of groups acting on trees with inversions. In Section 5, we apply the results of Section 4 to a tree product of groups and of quasi-HNN groups.

2. Groups acting on graphs. In this section, we begin by recalling some definitions taken from [3, 7]. First we give formal definitions related to groups acting on graphs with inversions. By a graph $X$ we understand a pair of disjoint sets $V(X)$ called the set of vertices and $E(X)$ called the set of edges, with $V(X)$ nonempty, equipped with two maps $E(X) \rightarrow V(X) \times V(X), y \rightarrow(o(y), t(y))$, and $E(X) \rightarrow E(X), y \rightarrow \bar{y}$, satisfying the conditions $\overline{\bar{y}}=y$ and $o(\bar{y})=t(y)$ for all $y \in E(X)$. The case $\bar{y}=y$ is possible for some $y \in E(X)$. For $y \in E(X), o(y)$ and $t(y)$ are called the ends of $y$ and $\bar{y}$ is called the inverse of $y$. There are obvious definitions of trees, morphisms of graphs, and $\operatorname{Aut}(X)$, the set of all automorphisms of the graph $X$ which is a group under the composition of morphisms. We say that a group $G$ acts on a graph $X$ if there is a group homomorphism $\phi: G \rightarrow \operatorname{Aut}(X)$. If $x \in X$ (vertex or edge) and $g \in G$, we write $g(x)$ for $(\phi(g))(x)$. Thus if $g \in G$ and $y \in E(X)$, then $g(o(y))=o(g(y)), g(t(y))=t(g(y))$, and $g(\bar{y})=\overline{g(y)}$. The case $g(y)=\bar{y}$ for some $g \in G$ and $y \in E(X)$ may occur. That is, $G$ acts with inversions on $X$.

We have the following definitions related to the action of the group $G$ on the graph $X$.

(1) If $x \in X$ (vertex or edge), define $G(x)$ to be the set $G(x)=\{g(x): g \in G\}$. This set is called the orbit that contains $x$. 
(2) If $x, y \in X$, define $G(x, y)$ to be the set $G(x, y)=\{g \in G: g(x)=y\}$, and $G(x, x)=G_{x}$, the stabilizer of $x$. Thus, $G(x, y) \neq \varnothing$ if and only if $x$ and $y$ are in the same orbit. If $y \in E(X)$ and $u \in\{o(y), t(y)\}$, then it is clear that $G_{\bar{y}}=G_{y}$ and $G_{y} \leq G_{u}$.

(3) If $X$ is connected, then a subtree $T$ of $X$ is called a tree of representatives for the action of the group $G$ on $X$ if $T$ contains exactly one vertex from each vertex orbit, and the subgraph $Y$ of $X$ containing $T$ is called a fundamental domain if each edge of $Y$ has at least one end in $T$, and $Y$ contains exactly one edge $y$ from each edge orbit such that $G(y, \bar{y})=\varnothing$, and exactly one pair $x, \bar{x}$ from each edge orbit such that $G(x, \bar{x}) \neq \varnothing$.

3. Notations. Let $G$ be a group acting on a tree $X$ with inversions, let $T$ be a tree of representatives for the action of $G$ on $X$, and let $Y$ be a fundamental domain. We have the following notations.

(1) For any vertex $v$ of $X$, let $v^{*}$ be the unique vertex of $T$ such that $G\left(v, v^{*}\right) \neq \varnothing$. That is, $v$ and $v^{*}$ are in the same vertex orbit.

(2) For each edge $y$ of $Y$, define the following:

(i) $[y]$ is an element of $G\left(t(y),(t(y))^{*}\right)$. That is, $[y]\left((t(y))^{*}\right)=t(y)$ is chosen as follows:

(a) if $o(y) \in V(T)$, then $[y]=1$ in case $y \in E(T)$, and $[y](y)=\bar{y}$ if $G(y, \bar{y}) \neq \varnothing$

(b) if $o(y) \notin V(T)$, then $[y]=[\bar{y}]^{-1}$ if $G(y, \bar{y})=\varnothing$, otherwise $[y]=[\bar{y}]$ if $G(y, \bar{y}) \neq \varnothing ;$

(ii) $-y$ is the edge $-y=[y]^{-1}(y)$ if $o(y) \in V(T)$, otherwise $-y=y$;

(iii) $+y$ is the edge $+y=[y](-y)$. It is clear that $t(-y)=(t(y))^{*}, o(+y)=$ $(o(y))^{*}, G_{-y} \leq G_{(t(y))^{*}}, \overline{(-y)}=+(\bar{y})$, and $G_{+y} \leq G_{(o(y))^{*}}$. Moreover, if $G(y$, $\bar{y}) \neq \varnothing$, or $y \in E(T)$, then $G_{-y}=G_{+y}=G_{y}$;

(iv) $\phi_{y}$ is the map $\phi_{y}: G_{-y} \rightarrow G_{+y}$ given by $\phi_{y}(g)=[y] g[y]^{-1}$;

(v) $\delta_{y}$ is the element $\delta_{y}=[y][\bar{y}]$. It is clear that $\phi_{y}$ is an isomorphism and $\delta_{y}=1$ if $G(y, \bar{y})=\varnothing$. Otherwise $\delta_{y}=[y]^{2}$.

4. On the intersection of vertex stabilizers of groups acting on trees with inversions. In this section, $G$ will be a group acting on a tree $X$ with inversions, $T$ is a tree of representatives for the action of $G$ on $X$, and $Y$ is a fundamental domain. We have the following definition.

DEFinition 4.1. A word $w$ of $G$ means an expression of the form $w=g_{0} \cdot y_{1} \cdot g_{1}$. $y_{2} \cdot g_{2} \cdots y_{n} \cdot g_{n}, n \geq 0, y_{i} \in E(Y)$, for $i=1, \ldots, n$, such that

(1) $g_{0} \in G_{\left(o\left(y_{1}\right)\right) *}$,

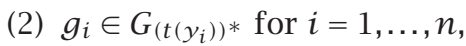

(3) $\left(t\left(y_{i}\right)\right)^{*}=\left(o\left(y_{i+1}\right)\right)^{*}$ for $i=1, \ldots, n-1$.

Define $o(w)=\left(o\left(y_{1}\right)\right)^{*}$ and $t(w)=\left(t\left(y_{n}\right)\right)^{*}$.

If $o(w)=t(w)$, then $w$ is called a closed word of $G$ of type $v, v=o(w)$.

The following concepts are related to the word $w$ defined above:

(i) $n$ is called the length of $w$ and is denoted by $|w|=n$, 
(ii) $w$ is called a trivial word of $G$ if $|w|=0$ (or $w=g_{0}$ ),

(iii) the value of $w$, denoted by [w], is defined to be the element of $G$ :

$$
[w]=g_{0}\left[y_{1}\right] g_{1}\left[y_{2}\right] g_{2} \cdots\left[y_{n}\right] g_{n}
$$

(iv) the inverse of $w$, denoted by $w^{-1}$, is defined to be the word of $G$ :

$$
w^{-1}=g_{n}^{-1} \cdot \bar{y}_{n} \cdot \delta_{y_{n}}^{-1} g_{n-1}^{-1} \cdots \cdots g_{2}^{-1} \cdot \bar{y}_{2} \cdot \delta_{y_{2}}^{-1} g_{1}^{-1} \cdot \bar{y}_{1} \cdot \delta_{y_{1}}^{-1} g_{0}^{-1},
$$

(v) $w$ is called reduced if $w$ contains no subword of the form $y_{i} \cdot g_{i} \cdot \bar{y}_{i}$ if $g_{i} \in G_{-y_{i}}$, or $y_{i} \cdot g_{i} \cdot y_{i}$ if $g_{i} \in G_{y_{i}}$ if $G\left(y_{i}, \bar{y}_{i}\right) \neq \varnothing$ for $i=1, \ldots, n$.

LEMMA 4.2. Let $w$ be a nontrivial reduced word of $G$ and let $a \in G_{o(w)}$ be such that $[w]^{-1} a[w] \in G_{[w](t(w))}$. Then there exists a reduced path $x_{1}, \ldots, x_{n}$ in $X$ from $o(w)$ to $[w](t(w))$ such that $a \in G_{x_{i}}$ for $i=1, \ldots, n$.

Proof. Let $w=g_{0} \cdot y_{1} \cdot g_{1} \cdot y_{2} \cdot g_{2} \cdots y_{n} \cdot g_{n}, n \geq 1$. By assumption, $[w]^{-1} a[w]=$ $b$, where $b \in G_{[w](t(w))}$. Consider the word

$$
\begin{aligned}
w_{0}= & g_{n}^{-1} \cdot \bar{y}_{n} \cdot \delta_{y_{n}}^{-1} g_{n-1}^{-1} \cdots g_{2}^{-1} \cdot \bar{y}_{2} \cdot \delta_{y_{2}}^{-1} g_{1}^{-1} \cdot \bar{y}_{1} \\
& \cdot \delta_{y_{1}}^{-1} g_{0}^{-1} a g_{0} \cdot y_{1} \cdot g_{1} \cdot y_{2} \cdot g_{2} \cdots y_{n} \cdot g_{n} b^{-1}
\end{aligned}
$$

Then $w_{0}$ is a nontrivial closed word of $G$ such that $\left[w_{0}\right]=1$, the identity element of $G$. Therefore by [4, Corollary 1$], w_{0}$ is not reduced. Since $w$ is reduced, then $w^{-1}$ is reduced. Therefore the only possibility that makes $w_{0}$ not reduced is $L_{i}^{-1} a L_{i} \in G_{-\left(\bar{y}_{i}\right)}=G_{-\left(\bar{y}_{i}\right)}=$ $G_{+y_{i}}$, where $L_{i}=g_{0}\left[y_{1}\right] g_{1}\left[y_{2}\right] g_{2} \cdots\left[y_{i-1}\right] g_{i-1}$ for $i=1, \ldots, n$ with the convention that $\left[y_{0}\right]=1$. Then $a \in L_{i} G_{+y_{i}} L_{i}^{-1}=G_{L_{i}\left(+y_{i}\right)}$ for $i=1, \ldots, n$. By taking $x_{i}=L_{i}\left(+y_{i}\right)$, we see that $a \in G_{x_{i}}$ for $i=1, \ldots, n$. By the corollary of [5, Theorem 1], $x_{1}, \ldots, x_{n}$ is a reduced path in $X$ from $o(w)$ to $[w](t(w))$. This completes the proof.

THEOREM 4.3. For any two vertices $u$ and $v$ of $X, G_{u}=G_{v}$ or $G_{u} \cap G_{v}$ is contained in $G_{x}$, where $x$ is an edge in the reduced path in $X$ joining $u$ and $v$.

Proof. If $G_{u}=G_{v}$, we are done. Let $G_{u} \neq G_{v}$ and $h \in G_{u} \cap G_{v}$. Then it is clear that $u \neq v$. We need to show that $h$ is in $G_{x}$, where $x$ is an edge in the reduced path in $X$ joining $u$ and $v$. We have $u=f\left(u^{*}\right)$ and $v=g\left(v^{*}\right)$, where $f$ and $g$ are in $G$ and $u^{*}$ and $v^{*}$ are the unique vertices of $T$ such that $G\left(u, u^{*}\right) \neq \varnothing$ and $G\left(v, v^{*}\right) \neq \varnothing$. Then $h=f a f^{-1}=g b g^{-1}$, where $a \in G_{u^{*}}$ and $b \in G_{v^{*}}$. By [5, Lemma 2], there exists a reduced word $w=g_{0} \cdot y_{1} \cdot g_{1} \cdot y_{2} \cdot g_{2} \cdots \cdot y_{n} \cdot g_{n}$ of $G$ such that $o(w)=u, t(w)=v$, and $[w]=g \cdot w$ is nontrivial. For, if $w$ is trivial, then $u^{*}=v^{*}$ and $f^{-1} g \in G_{u^{*}}$. This implies that $f^{-1} g\left(v^{*}\right)=u^{*}$, or equivalently $u=v$. This contradicts the assumption that $u \neq v$. By Lemma 4.2, there exists a reduced path $p_{1}, \ldots, p_{n}$ in $X$ joining $o(w)=u^{*}$ and $[w](t(w))=f^{-1} g\left(v^{*}\right)$ such that $a \in G_{p_{i}}$ for $i=1, \ldots, n$. Let $x_{i}=f\left(p_{i}\right), i=1, \ldots, n$. Then it is clear that $x_{1}, \ldots, x_{n}$ is the reduced path in $X$ joining $u$ and $v$ and $h \in G_{x_{i}}$ for $i=1, \ldots, n$. This implies that $G_{u} \cap G_{v} \leq G_{x_{i}}$ for $i=1, \ldots, n$. This completes the proof. 
We have the following corollaries of Theorem 4.3.

Corollary 4.4. For any edge $x$ of $X, G_{o(x)}=G_{t(x)}$ or $G_{o(x)} \cap G_{t(x)}=G_{x}$.

COROLlary 4.5. Let $u$ and $v$ be two vertices of $X$ and let $x_{1}, \ldots, x_{n}$ be the reduced path in $X$ joining $u$ and $v$ such that $G_{u} \neq G_{v}$. Then $G_{u} \cap G_{v} \leq I_{i=1}^{n} G_{x_{i}}$.

COROLlary 4.6. Let $u$ and $v$ be two vertices of $X$ such that $G_{u} \neq G_{v}$ and let $x$ be an edge in the reduced path in $X$ joining $u$ and $v$. Then $G_{u} \cap G_{v} \leq G_{X}$.

Corollary 4.7. Let $u$ be a vertex of $X$ and let $v$ be a vertex of $T$. Then $G_{u} \cap G_{v} \leq G_{x}$, where $x$ is an edge in the reduced path in $X$ joining $u$ and $v$, or $u^{*}=v$ and $G_{u} \cap G_{v}=G_{v}$.

COROLlary 4.8. Let $u$ be a vertex of $X$. Then $G_{u} \cap G_{u^{*}} \leq G_{x}$, where $x$ is an edge in the reduced path in $X$ joining $u$ and $u^{*}$, or $u^{*}=u$ and $G_{u} \cap G_{u^{*}}=G_{u}$.

Corollary 4.9. For any edge $y$ of $Y, G_{(o(y)) *}=G_{(t(y)) *}$, or $G_{(o(y)) *} \cap G_{(t(y)) *} \leq G_{m}$, where $m$ is an edge in the reduced path in $T$ joining $(o(y))^{*}$ and $(t(y))^{*}$.

5. Applications. In this section Theorem 4.3 and its corollaries are applied to a nontrivial tree product of groups introduced in [1] and of quasi-HNN groups introduced in [2].

In [5, Lemma 8], Mahmood showed that if $G=\prod_{i \in I}^{*}\left(A_{i}, U_{j k}=U_{k j}\right)$ is a nontrivial tree product of the groups $A_{i}, i \in I$, then there exists a tree $X$ on which $G$ acts without inversions such that any tree of representatives for the action of $G$ on $X$ equals the fundamental domain and for every vertex $u$ of $X$ and every edge $x$ of $X, G_{u}$ is a conjugate of $A_{i}$ for some $i$ in $I$ and $G_{X}$ is a conjugate of $U_{i k}$ for some $i, k$ in $I$.

In [6, Lemma 5.1], Mahmood and Khanfar showed that if $G^{*}$ is the quasi-HNN group $G^{*}=\left\langle G, t_{i}, t_{j} \mid \operatorname{rel} G, t_{i} A_{i} t_{i}^{-1}=B_{i}, t_{j} C_{j} t_{j}^{-1}=C_{j}, t_{j}^{2}=c_{j}, i \in I, j \in J\right\rangle$, then there exists a tree $X$ on which $G^{*}$ acts with inversions such that $G^{*}$ is transitive on $V(X)$ and for every vertex $v$ of $X$ and every edge $x$ of $X, G_{v}^{*}$ is a conjugate of $G$ and $G_{x}^{*}$ is a conjugate of $A_{i}, i \in I$, or a conjugate of $C_{j}, j \in J$.

Then by Theorem 4.3, the following two propositions hold.

Proposition 5.1. Let $G=\prod_{i \in I}^{*}\left(A_{i}, U_{j k}=U_{k j}\right)$ be a nontrivial tree product of the groups $A_{i}, i \in J$. Then for any $g$ in $G$ and $i$ and $s$ in $I$, either $g A_{i} g^{-1} \cap A_{s}$ is contained in a conjugate of $U_{j k}$ or $i=j, g \in A_{i}$, and $g A_{i} g^{-1} \cap A_{i}=A_{i}$. Moreover, if $A_{i}$ and $A_{j}$ are adjacent, then $A_{i} \cap A_{j}=U_{i j}$.

Proposition 5.2. Let $G^{*}$ be the quasi-HNN group

$$
G^{*}=\left\langle G, t_{i}, t_{j} \mid \operatorname{rel} G, t_{i} A_{i} t_{i}^{-1}=B_{i}, t_{j} C_{j} t_{j}^{-1}=C_{j}, t_{j}^{2}=c_{j}, i \in I, j \in J\right\rangle .
$$

Then for any $g \in G^{*}, g G g^{-1} \cap G$ is contained either in a conjugate of $A_{i}, i \in I$, or in a conjugate of $C_{j}, j \in J$, or $g \in G$ and $g G g^{-1} \cap G=G$.

REMARK 5.3. If $J=\varnothing$, then $G^{*}$ is the HNN group $G^{*}=\left\langle G, t_{i}\right| \operatorname{rel} G, t_{i} A_{i} t_{i}^{-1}=B_{i}, i \in$ $I\rangle$. Then, for any $g \in G^{*}$, either $g G g^{-1} \cap G$ is contained in a conjugate $A_{i}, i \in I$, or $g \in G$ and $g G g^{-1} \cap G=G$. 
ACKNOWLEDGMENT. The author would like to thank the referees for their sincere evaluation and constructive comments which improved the paper considerably.

\section{REFERENCES}

[1] A. Karrass and D. Solitar, The subgroups of a free product of two groups with an amalgamated subgroup, Trans. Amer. Math. Soc. 150 (1970), 227-255.

[2] M. I. Khanfar and R. M. S. Mahmood, On quasi HNN groups, Kuwait J. Sci. Engrg. 29 (2002), no. 2, 13-24.

[3] R. M. S. Mahmood, Presentation of groups acting on trees with inversions, Proc. Roy. Soc. Edinburgh Sect. A 113 (1989), no. 3-4, 235-241.

[4] _ The normal form theorem of groups acting on trees with inversions, J. Univ. Kuwait Sci. 18 (1991), 7-16.

[5] _ The subgroup theorem for groups acting on trees, Kuwait J. Sci. Engrg. 25 (1998), no. 1, 17-33.

[6] R. M. S. Mahmood and M. I. Khanfar, Subgroups of quasi-HNN groups, Int. J. Math. Math. Sci. 31 (2002), no. 12, 731-743.

[7] J.-P. Serre, Arbres, Amalgames, SL2, Société Mathématique de France, Paris, 1977.

[8] C. T. C. Wall, Homological Group Theory, London Mathematical Society Lecture Note Series, vol. 36, Cambridge University Press, Cambridge, 1979.

R. M. S. Mahmood: Ajman University of Science and Technology, P.O. Box 5102, Abu Dhabi, UAE

E-mail address: rasheedmsm@yahoo.com 


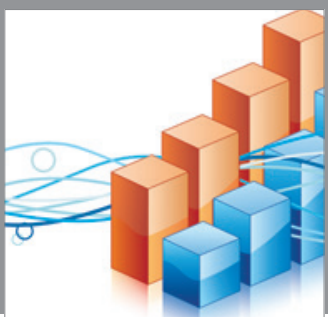

Advances in

Operations Research

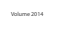

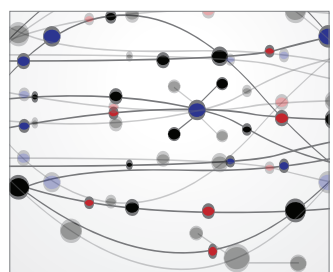

\section{The Scientific} World Journal
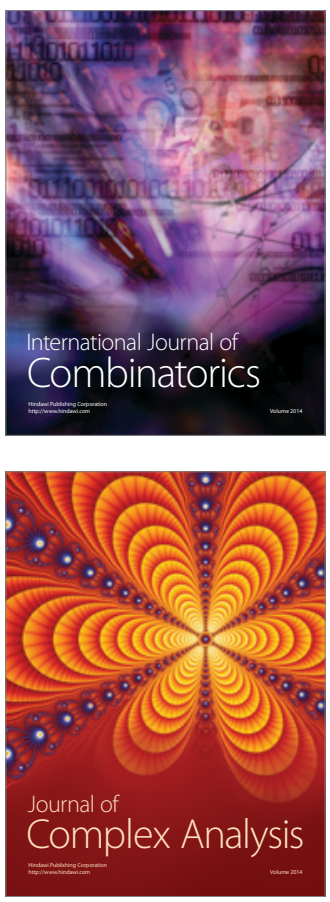

International Journal of

Mathematics and

Mathematical

Sciences
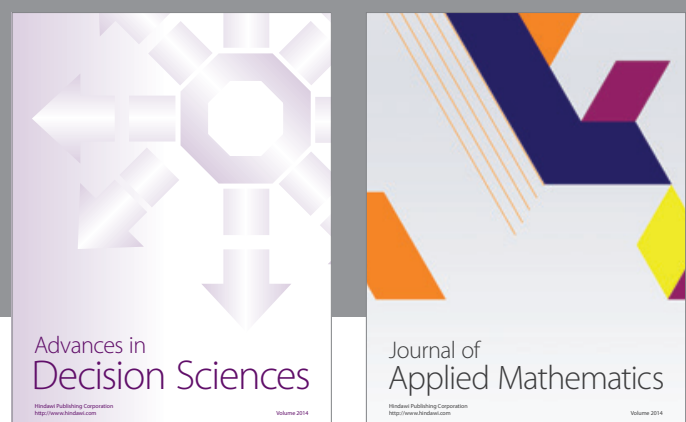

Journal of

Applied Mathematics
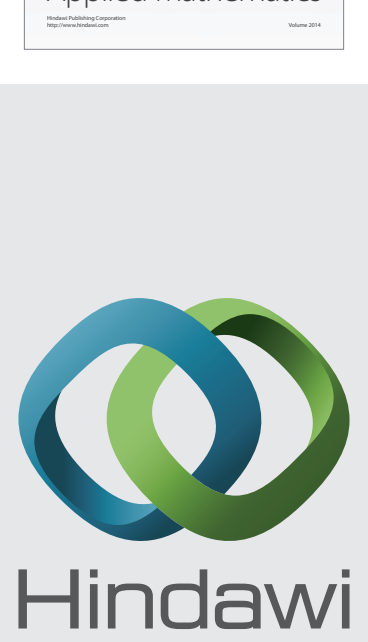

Submit your manuscripts at http://www.hindawi.com
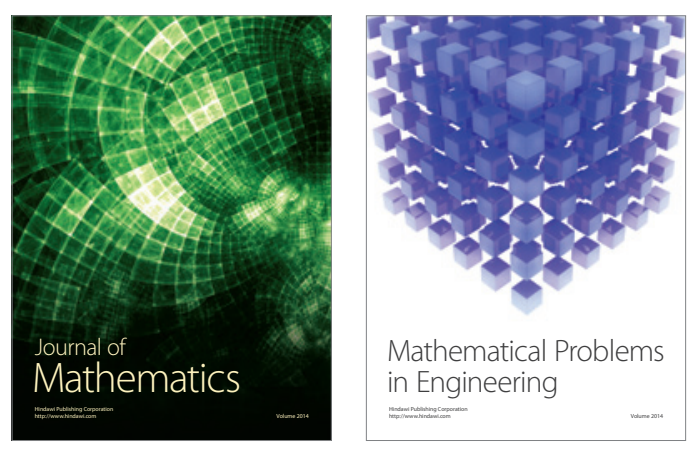

Mathematical Problems in Engineering
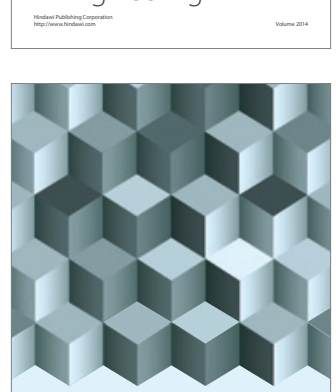

Journal of

Function Spaces
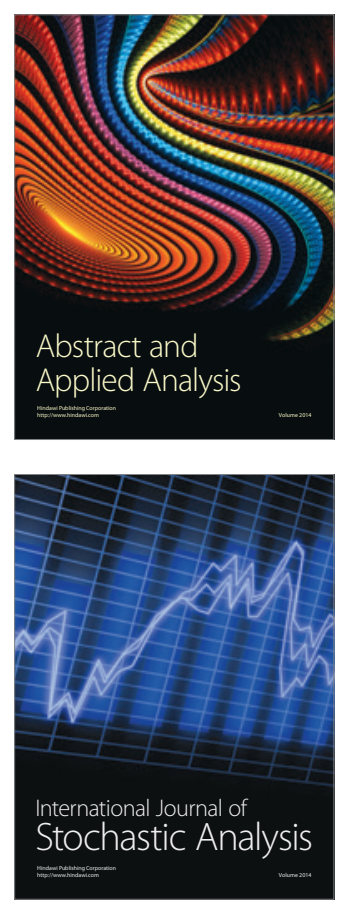

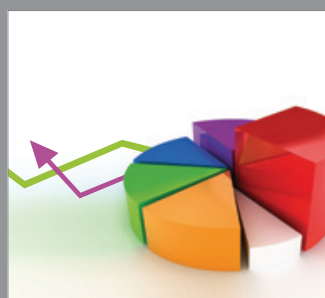

ournal of

Probability and Statistics

Promensencen
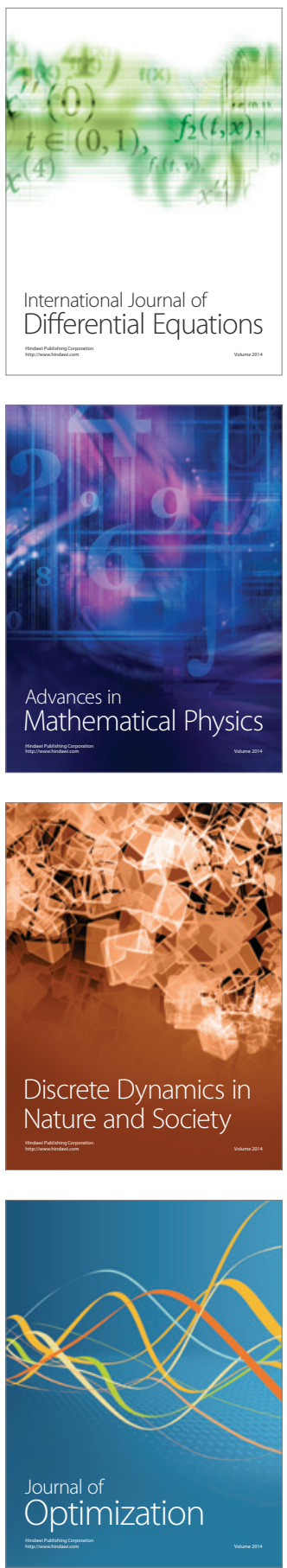